\title{
Structural, electrical conductivity and dielectric relaxation behavior of LiHf2(PO4)3 ceramic powders
}

\begin{abstract}
Lithium hafnium phosphate LiHf2(PO4)3 (LHP) was synthesized via solid-state synthesis technique. The sintering behavior, structure, and phase composition of the as-prepared sample was analyzed using X-ray diffraction (XRD) characterization technique. The XRDRietveld refinement analysis showed that after sintering at low temperatures 500 to $1000{ }^{\circ} \mathrm{C}$, it exhibited various secondary phases. However, a single phase was observed as the sintering temperature increases from 1100 to $1200{ }^{\circ} \mathrm{C}$. LHP sintered at $1100{ }^{\circ} \mathrm{C}$ produced real features of sodium superionic conductor type (NASICON-type) with hexagonal crystal axis indicating $\mathrm{R}-3 \mathrm{c}$ space group. The electrical properties were studied using impedance spectroscopy technique. Frequency and temperature dependence behavior of conductivity (ac and dc) and dielectric permittivity were studied. The results obtained describes the conduction mechanism in the system. Electric modulus formalism was performed to investigate the relaxation behavior which showed that as measuring temperature increases, the relaxation frequency increases whereas relaxation time decreases. This behavior explains the hopping mechanism of the charge carriers in the system. Likewise, the correlated barrier hopping model elucidates the dominant hopping mechanism.
\end{abstract}

Keyword: LiHf2(PO4)3 ceramics; NASICON structure; Conductivity; Dielectric relaxation properties 
